\title{
Evapotranspiração e coeficiente de cultura da melancia na microrregião de Teresina, PI, Brasil
}

\author{
Valber Mendes Ferreira ${ }^{1 *}$, Antonio Evaldo Klar' ${ }^{1}$ Aderson Soares Andrade Júnior², \\ Edson Alves Bastos², Simone Raquel Mendes Oliveira ${ }^{3}$ \\ 'Universidade Estadual Paulista Júlio de Mesquita Filho, Botucatu, SP, Brasil \\ ${ }^{2}$ Embrapa Meio Norte, Teresina, PI, Brasil \\ ${ }^{3}$ Universidade Federal do Piauí, Teresina, Pl, Brasi \\ *Autor correspondente, e-mail: burite@hotmail.com
}

\section{Resumo}

A determinação dos valores do coeficiente de cultura (Kc), durante o ciclo de desenvolvimento, é fundamental para se obter as necessidades hídricas de uma cultura. O presente trabalho teve por objetivo determinar a evapotranspiração e o Kc da melancia, cultivares Shadow, Top Gun e Crimson Sweet, durante os diferentes estádios de desenvolvimento utilizando o método de balanço de água no solo. O experimento foi conduzido no campo experimental da Embrapa Meio Norte, em Teresina, Pl, durante o período de agosto a outubro de 2010. A evapotranspiração de referência foi estimada pelo método de Penman-Monteith com base nos dados meteorológicos obtidos por uma estação agrometeorologia automática. Os resultados mostram que a ETc foi de $225,4 \mathrm{~mm}$ para todo o ciclo da cultura, correspondente a um valor médio de $3,8 \mathrm{~mm} \mathrm{~d}^{-1}$. O estádio fenológico de maior demanda hídrica foi o intermediário (reprodutivo) com $5,2 \mathrm{~mm} \mathrm{~d}^{-1}$. Os valores de $\mathrm{Kc}$ foram 0,39; 0,80; 1, 14; 0,59 (Crimson Sweet); 0,35; 0,72; 1,20; 0,30 (Top Gun) e 0,34; 0,58; 0,89; 0,55 (Shadow), para as fases inicial, crescimento, intermediário e final, respectivamente.

Palavras-chave: manejo de irrigação, consumo hídrico, Kc, Citrullus lanatus

\section{Watermelon evapotranspiration and crop coefficient in Teresina microregion - Piaui, Brazil}

\begin{abstract}
The determination of crop coefficient $(\mathrm{KC})$ values during the crop development cycle is essential to obtain the water requirements for the culture. The present study aimed to determine the evapotraspiration and Kc on Shadow, Top Gun and Crimson Sweet watermelon cultivars, during different stages of development using the method of soil water balance. The experiment was conducted at Embrapa Meio Norte in Teresina, Pl, experimental field, from August to October, 2010. The evapotranspiration reference was estimated by Penman-Monteith, based on meteorological data obtained by an automatic weather station. According to the results, the ETc was $224.4 \mathrm{~mm}$, corresponding to an average of $3.8 \mathrm{~mm}$ day $^{-1}$. The higher water demand was $5.5 \mathrm{~mm} \mathrm{day}^{-1}$, obtained at the intermediate phenological stage. The Kc values were $0.39,0.80,1.14,0.59$ (Crimson Sweet), $0.35,0.72,1.20,0.30$ (Top gun) and 0.34, 0.58, 0.89, 0.55 (Shadow), for initial, growth, intermediate and final phases, respectively.
\end{abstract}

Keywords: Citrullus lanatus, irrigation management, Kc, water consumption 


\section{Introdução}

A melancia (Citrullus lanatus) pertence à família das cucurbitáceas e tem grande expressão econômica e social, possuindo propriedades nutricionais e terapêuticas (Dias et al., 2006).

Segundo O IBGE (2010), em termos de Brasil, foram produzidas 2.052.928 † de melancia em uma área plantada de 96.477 ha, sendo que as Regiões Nordeste e Sul são as principais produtoras com respectivamente $34,15 \%$ e $24,64 \%$ do total da produção nacional dessa olerícola. No Nordeste, Bahia, Pernambuco e Rio Grande do Norte são responsáveis por $59 \%$ da produção nacional, enquanto que o Piauí ocupa a $4^{\circ}$ posição, com uma produção de 67.023 † em uma área plantada de 2.545 ha.

O estado do Piauí caracteriza-se por apresentar solos de textura arenosa, possuir considerável potencial hídrico de boa qualidade e clima favorávelà produção de frutas e hortaliças sob condições de irrigação. Como em todo - Nordeste brasileiro, as condições climáticas favoráveis ao cultivo de melancia, clima seco e quente, têm elevado consideravelmente a produção de frutos de excelente qualidade. No entanto, existem poucas pesquisas no estado do Piauí sobre o consumo hídrico da melancia e como conseqüência de carências de informações a falta do manejo racional da irrigação a aplicação da lâmina de irrigação tem sido feito de maneira aleatória.

O conhecimento do coeficiente de cultura (Kc) é fundamental para se outorgar, dimensionar e manejar a irrigação de uma cultura e, uma vez que esses valores variam conforme a disponibilidade energética do local, variedade e idade da planta, é importante à obtenção de valores regionalizados (Silva et al., 2006).

Já existem recomendações de Kc para a melancia no Piauí (Bastos et al., 2007; Ferreira, 2010), no Ceará (Carvalho et al., 2007; Miranda et al., 2004; Bezerra \& Oliveira, 1999), mas ainda não há nenhuma recomendação para a microrregião de Teresina-PI para as cultivares Shadow, Top Gun e Crimson Sweet.

Este trabalho teve por objetivo determinar o Kc da melancia nos diferentes estádios fenológicos, utilizando o balanço hídrico de água no solo, visando o manejo racional da irrigação na microrregião de Teresina, PI.

\section{Material e Métodos}

Local e característica da área experimental O experimento foi conduzido no campo experimental da Embrapa Meio-Norte, no município de Teresina, PI $\left(05^{\circ} 05^{\prime} \mathrm{S} ; 42^{\circ} 48^{\prime} \mathrm{W}\right.$ e 74,4 m). O clima do município, de acordo com a classificação climática de Thornthwaite \& Mather (1955) é ClsA'a', caracterizado como subúmido seco, megatérmico, com excedente hídrico moderado no verão. O solo foi classificado como um ARGISSOLO VERMELHO AMARELO eutrófico (Santos et al., 2006) com algumas características químicas apresentadas na Tabela 1.

Segundo Carlos (2002), os frutos do cultivar Crimson sweet tem formato arredondado, casca clara com estrias verdeescuro, polpa vermelho intenso muito doce, sendo os frutos de tamanhos médio e grande de melhor qualidade.

O híbrido Top Gun é uma planta vigorosa de fruto redondo-ovalado, casca verde escura, com estrias verdes claro, polpa vermelha brilhante com excelente textura e peso médio de 10 a $12 \mathrm{~kg}$. Seu ciclo varia de acordo com o local de cultivo, normalmente em torno de 90 a 95 dias (Syngenta Seeds, 2010).

A melancia híbrida triplóide Shadow destaca-se por ser uma triplóide (sem sementes). É uma planta com excelente vigor, possui fruto redondo-ovalado, casca verde escura, polpa vermelha e com peso médio de 7 a $8 \mathrm{~kg}$ (Syngenta Seeds, 2010).

O preparo do solo constou de uma aração e duas gradagens, o que foi suficiente

Tabela 1. Características químicas do solo da área experimental.

\begin{tabular}{|c|c|c|c|c|c|c|c|c|c|c|c|}
\hline \multirow{2}{*}{ Camadas (m) } & \multirow{2}{*}{$\mathrm{pH}\left(\mathrm{H}_{2} \mathrm{O}\right)$} & $\mathrm{P}$ & $K$ & $\mathrm{Ca}^{2+}$ & $\mathrm{Mg}^{2+}$ & $\mathrm{Na}^{+}$ & $\mathrm{Al}^{3+}$ & $S$ & CTC & \multirow{2}{*}{$\begin{array}{c}V \\
(\%)\end{array}$} & \multirow{2}{*}{$\begin{array}{c}\mathrm{MO} \\
\left(\mathrm{g} / \mathrm{kg}^{-1}\right)\end{array}$} \\
\hline & & \multicolumn{3}{|c|}{$\mathrm{mg} / \mathrm{dm}^{-3}$} & \multicolumn{3}{|c|}{$\mathrm{mmol} / \mathrm{dm}^{-3}$} & & & & \\
\hline $0,0-0,20$ & 5,35 & 54,34 & 1,8 & 14,9 & 6,2 & 0,5 & 0,5 & 23,4 & 4,26 & 54,87 & 6,00 \\
\hline $0,20-0,40$ & 4,51 & 11,84 & 1,1 & 11,4 & 6.3 & 0,1 & 4,9 & 18,9 & 5,67 & 33,33 & 4,72 \\
\hline
\end{tabular}


para um adequado destorroamento do solo. A semeadura foi realizada em ambiente protegido, no dia 20/08/2010. Utilizaram-se bandejas de 128 células com substrato composto para produção de mudas. Após 12 dias transcorridos (01/09/2010), foram transplantadas para o campo, com espaçamento de 2,0 m entre fileiras e 1,0 m entre plantas. A adubação foi toda via fertirrigação com bombas injetoras de fertilizante do tipo TMB, instaladas em cavaletes nas linhas de derivação. As quantidades dos fertilizantes foram aplicadas a partir da marcha de absorção de nutrientes pela cultura da melancia (Sousa et al., 1999).

Irrigação

A aplicação de água foi realizada por um sistema de irrigação por gotejamento. Utilizou-se o tubo gotejador S.Typhoon 1650, autocompensante com vazão de 1,65 L.h ${ }^{-1}$, espaçamento entre gotejadores de $0,50 \mathrm{~m}$. A linha lateral possuía diâmetro de $50 \mathrm{~mm}$ e comprimento de $12 \mathrm{~m}$. Essa linha era conectada a uma linha principal, com diâmetro $75 \mathrm{~mm}$. O sistema de irrigação foi dividido em cinco setores correspondente a cinco lâminas $(L 1=50, L 2=75$, $L 3=100, L 4=125, L 5=150 \%$ da evapotranspiração de referência) e a irrigação de cada setor foi independente, controlada com um registro de esfera e hidrômetro, para quantificação da lâmina de irrigação aplicada. No entanto, para o referido estudo admitiu-se somente a $\mathrm{L} 3=100 \%$ da evapotranspiração de referência.

Os valores de conteúdo de água no perfil do solo (\%, em volume) foram obtidos utilizando-se uma sonda FDR, modelo Diviner $2000^{\circledR}$.

As leituras de frequência relativa (FR) eram efetuadas diariamente, sempre antes e aproximadamente 24 horas após as irrigações. Para tanto, foram instalados 30 tubos de acesso em PVC, com 1,0 m de comprimento, na direção perpendicular à linha de gotejo entre duas plantas. Instalaram-se seis tubos por faixa de irrigação, sendo dois em parcelas distintas de cada cultivar. O conteúdo médio de água no solo, em cada camada, foi calculado pela média dos valores de umidade medidos em dois tubos de acesso.
As profundidades das leituras foram de 10, 20, 30, 40, 50, 60, $70 \mathrm{~cm}$. Contudo, no cálculo do balanço hídrico foram considerados os valores de conteúdo de água no solo, resultantes do somatório do conteúdo de água no solo nas camadas de 0 a $40 \mathrm{~cm}$.

Estimativa da evapotranspiração da cultura (ETC)

A evapotranspiração da cultura foi estimada utilizando-se uma simplificação do método do balanço de água no solo.

No cálculo do balanço hídrico, admitiuse a drenagem como nula. Os fluxos ascendentes e descendentes no solo não foram admitidos, uma vez que não houve variação nos valores diários de umidade no solo abaixo da camada controle do solo $(40 \mathrm{~cm})$.

A ETr da lâmina onde se fez a reposição de $100 \%$ da ETo acumulada no período, admitida como sendo a ETC.

$$
E T r_{i j}=\sum_{z=10}^{40} \theta_{(i j)}-\sum_{z=10}^{40} \theta_{(i+1) j} \ldots . .(1)
$$

Em que

ETrij - Evapotranspiração real da cultura, no dia i, lâmina j (mm);

(ij) - Conteúdo de água no solo (\% em volume), no dia i, lâmina j;

$(i+1) j$ - Conteúdo de água no solo (\% em volume) no dia seguinte 1 (i+1), lâmina j.

Z - profundidade do solo na zona de raízes, para a cultura da melancia $(40 \mathrm{~cm})$.

Estimativa dos coeficientes de cultura $(\mathrm{Kc})$

O coeficiente de cultura $(\mathrm{KC})$ foi obtido pela relação entre a evapotranspiração da cultura (ETC), calculada pelo método do balanço hídrico no solo e a evapotranspiração de referência (ETo), estimada pelo método de Penman - Monteith.

$$
K C=\frac{E T C}{E T O}
$$

Em que:

ETc - evapotranspiração da cultura $\left(\mathrm{mm} \cdot \mathrm{dia}^{-1}\right)$; ETo - evapotranspiração de referência $\left(\mathrm{mm} \cdot \mathrm{dia}^{-1}\right)$.

\section{Resultados e Discussão}

De maneira geral, pela Tabela 2, observa-se que no período de cultivo da 
melancia a temperatura média do ar foi de $29,5^{\circ} \mathrm{C}$ dentro da faixa de valores considerados adequados para o desenvolvimento da cultura. Segundo Rezende et al. (2010) as cucurbitáceas se adaptam bem às zonas quentes e semiáridas, com alta luminosidade e temperaturas do ar entre $18^{\circ} \mathrm{C}$ a $30^{\circ} \mathrm{C}$, não tolerando temperaturas abaixo de $10^{\circ} \mathrm{C}$. Os eventos de precipitação concentraram-se mais nos últimos dias do mês de outubro, provocando maiores variações no conteúdo de água no solo, já na fase final do ciclo.

A evapotranspiração de referência média diária variou de 4,1 a $5,6 \mathrm{~mm} \mathrm{dia}^{-1}$, no mês de outubro e 5,2 a 5,7 $\mathrm{mm} \mathrm{dia}^{-1}$, no mês de setembro (Tabela 2). Observa-se que a ocorrência de chuvas concentradas no mês de outubro teve efeito de redução nos valores de ETo, devido ao aumento da nebulosidade na atmosfera, comprovado pela redução na radiação solar global. Historicamente, os valores máximos diários de ETo na região ocorrem no mês de outubro (Andrade Júnior et al., 2004), comportamento esse que foi alterado nesse ano específico face à ocorrência de chuvas durante esse mês.

Tabela 2. Valores médios mensais de precipitação pluviométrica (PP), temperatura do ar - média (Tmed), evapotranspiração de referência (ETo) estimada pelo método de Penman-Monteith, umidade relativa do ar média (URmed), velocidade do vento a $2 \mathrm{~m}$ de altura ( $V \mathrm{~V})$, saldo da radiação solar (RS), referentes ao período de setembro a outubro de 2010.

\begin{tabular}{|c|c|c|c|c|c|c|c|}
\hline MÊS & Dia & $\begin{array}{c}\mathrm{PP} \\
(\mathrm{mm})\end{array}$ & $\begin{array}{c}\text { Tmed } \\
\left({ }^{\circ} \mathrm{C}\right)\end{array}$ & $\begin{array}{c}\text { ETo } \\
\left(\mathrm{mm} \mathrm{dia}^{-1}\right)\end{array}$ & $\begin{array}{l}\text { UR } \\
(\%)\end{array}$ & $\begin{array}{c}\mathrm{Vv} \\
\left(\mathrm{m} \cdot \mathrm{s}^{-1}\right)\end{array}$ & $\begin{array}{c}\text { RS } \\
\left(\mathrm{MJ} \mathrm{m}^{-2} \mathrm{dia}^{-1}\right)\end{array}$ \\
\hline \multirow{3}{*}{ Setembro } & 1 a 10 & 0,3 & 28,4 & 5,2 & 54,3 & 0,9 & 22,4 \\
\hline & 11 a 20 & 1,4 & 29,5 & 5,7 & 51,5 & 0,9 & 24,1 \\
\hline & 21 a 30 & 0,0 & 30,0 & 5,7 & 51,7 & 1,0 & 24,3 \\
\hline \multirow{3}{*}{ Outubro } & 1 a 10 & 0,0 & 30,3 & 5,6 & 54,9 & 1,0 & 23,8 \\
\hline & 11 a 20 & 0,0 & 30,4 & 5,4 & 54,2 & 1,0 & 22,9 \\
\hline & 21 a 30 & 24,3 & 28,4 & 4,1 & 70,8 & 0,9 & 19,7 \\
\hline \multicolumn{2}{|c|}{ Med/Tot. } & 4,3 & 29,5 & 5,3 & 56,2 & 1,0 & 22,9 \\
\hline
\end{tabular}

As variações na evapotranspiração diária da cultura (ETc) e a evapotranspiração de referência (ETo) são mostradas na Figura 1. Observa-se que a ETc foi aproximadamente inferior que a ETo nos primeiros 41 dias após transplantio (DAT). Entre o $41^{\circ}$ e $50^{\circ}$ dias os valores são próximos, coincidindo com a fase de frutificação, atingindo valores máximos em torno de $5,2 \mathrm{~mm} \mathrm{dia}^{-1}$. Do $51^{\circ}$ ao $60^{\circ}$ dia, observa-se uma queda nos valores de evapotranspiração provocado pela nebulosidade ocorrida nesse período.

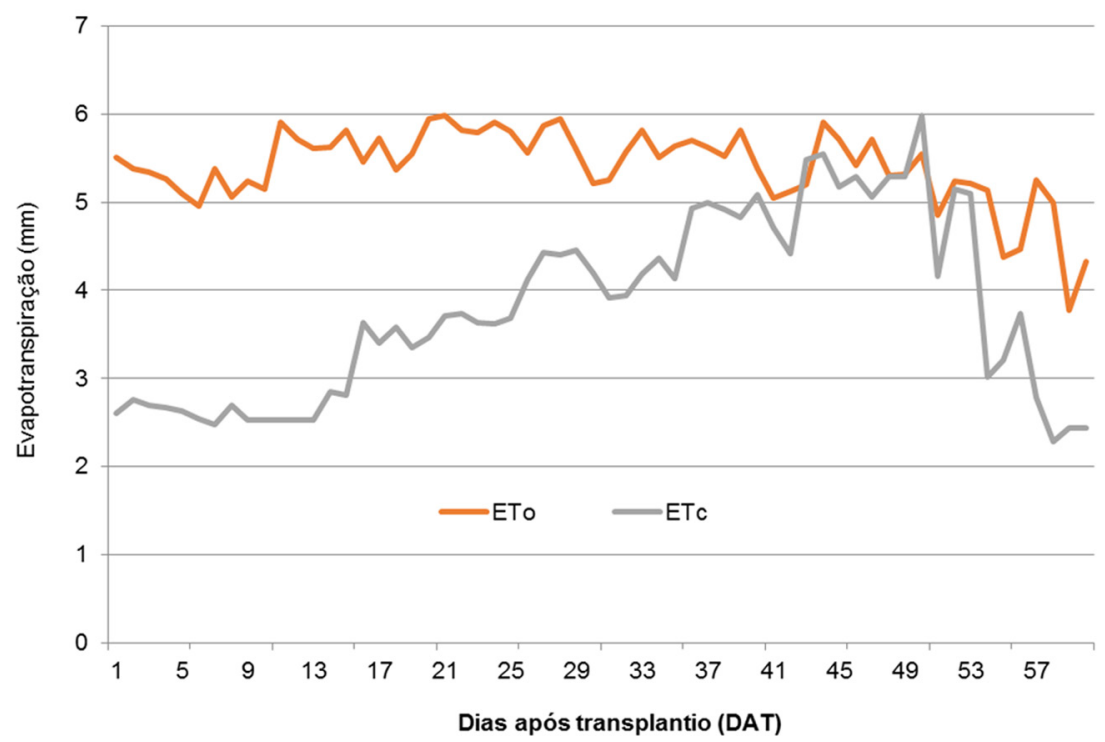

Figura 1. Valores médios de evapotranspiração da melancia comparados com evapotranspiração de referência. 
Na Tabela 3, são apresentados os valores totais de evapotranspiração da melancia (ETC) e de referência (ETo) e os coeficientes de cultivo (KC) em cada estádio fonológico prara cada cultivar.

Constata-se que os valores medidos da evapotranspiração da cultura (ETC) variam de acordo com o seu estádio fenológico (o que já era esperado). De maneira geral, verificase que a ETc $(225,4 \mathrm{~mm})$ foi inferior a ETo $(317,6$ $\mathrm{mm})$, sendo que a maior amplitude entre ambas encontrada no estádio inicial $(35,9 \mathrm{~mm})$ e a menor no estádio final $(9,9 \mathrm{~mm})$. Para a cultura da melancia na região Nordeste o valor obtido para a ETc $\left(225,4\right.$ ou 3,8 $\left.\mathrm{mm} \mathrm{dia}^{-1}\right)$ está inferior aos valores obtidos por Carvalho et al. (2007) e Miranda et al. (2004).

A variação do Kc durante to do o ciclo das cultivares é mostrada na Figura 2. O coeficiente de cultura medido para as variedades Crimson Sweet, Top Gun e Shadow apresentaram valores mínimos de 0,39, 0,35 e 0,38 respectivamente nos primeiros 20 DAT.

Tabela 3. Duração (dias) dos estádios fonológicos da cultura, evapotranspiração da cultura (ETc) e referência (ETo) e valores de Kc médios para cada estádio e cultivares.

\begin{tabular}{lccccccc}
\hline \multirow{2}{*}{ Estádios } & $\begin{array}{c}\text { Duração } \\
\text { (dias) }\end{array}$ & $\begin{array}{c}\text { ETc } \\
(\mathrm{mm})\end{array}$ & $\begin{array}{c}\text { ETo } \\
(\mathrm{mm})\end{array}$ & Crimson Sweet & Top Gun & Shadow \\
\cline { 7 - 8 } & 13 & 33,7 & 69,6 & 0,39 & 0,35 & 0,34 \\
Inicial & 15 & 54,5 & 86,2 & 0,80 & 0,72 & 0,58 \\
Crescimento & 22 & 106,2 & 120,9 & 1,14 & 1,20 & 0,89 \\
Intermediário & 10 & 31,0 & 40,9 & 0,59 & 0,30 & 0,55 \\
\hline Final & 60 & 225,4 & 317,6 & - & - & - \\
\hline Total & & & & & & \\
\hline
\end{tabular}

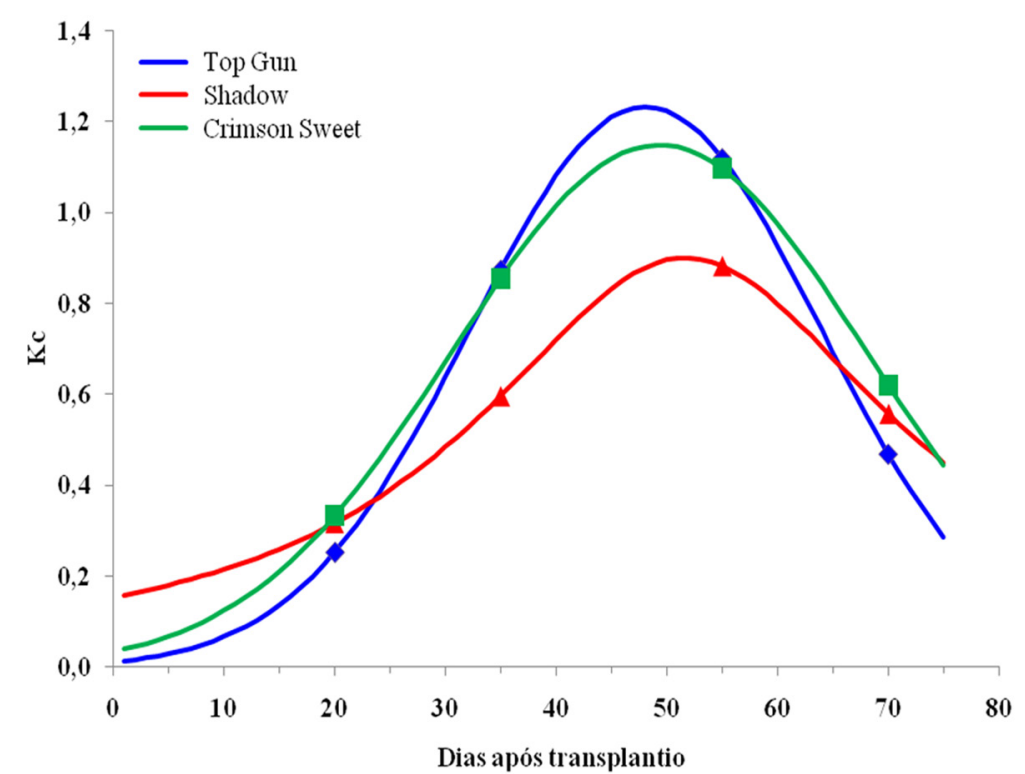

Figura 2. Evolução do coeficiente de cultivo $(K C)$ determinado ao longo do ciclo das variedades de melancia Crimson Sweet, Top Gun e Shadow.

Com o desenvolvimento da cultura, o Kc aumentou, alcançando o valor máximo de 1,14; 1,20 e 0,89 aos 55 DAT, coincidindo com o período da frutificação. A partir desse pico, verificou-se um decréscimo, próprio da senescência das cultivares.

Os coeficientes de cultivo determinados neste estudo foram superiores aos recomendados pela FAO (Allen et al., 2006) para as fases inicial e intermediária, tanto para a ETo determinada em lisímetros, quanto para a ETo estimada pelo método FAO Penman-Monteith.

Valores maiores de Kc foram observados por Bezerra \& Oliveira (1999), que pesquisando a cultivar de melancia Crimson Sweet, obtiveram valor máximo de 1,27 em torno dos 45 DAP, no período do inicio da frutificação. Valores maiores ao da pesquisa também foram encontrados 
por Bastos et al. (2007), que trabalhando com a cultivar Crimson Sweet irrigada por gotejamento nas condições de solo e clima dos Tabuleiros Litorâneos do Piauí, apresentou valores de Kc de 1,3 no estádio intermediário e 0,43 no estádio final. Já Miranda et al. (2004) utilizando os valores de ETo estimados pelo método Penman Monteith obtiveram valor máximo de Kc similar ao desta pesquisa para a cultivar Crimson Sweet. O valor determinado foi 1,15 para o período da frutificação.

Os maiores valores de Kc obtidos no trabalho para as variedades Crimson sweet e Top Gun foram superiores e para a variedade Shadow foram inferiores que os obtidos por Carvalho et al. (2007), nos estágios intermediários. Estas diferenças podem ser explicadas pelas características dos híbridos e pelas diferentes condições de solo e clima (umidade relativa do ar, temperatura), durante a execução do experimento. Silva et al. (2006) já relatavam a diferença dos valores de Kc's quanto a disponibilidade energética do local, tipo de solo, variedade e idade da planta.

\section{Conclusões}

A evapotranspiração da melancia foi de $225,4 \mathrm{~mm}$ para todo o ciclo da cultura, correspondente a um valor médio de $3,8 \mathrm{~mm}$ $\mathrm{dia}^{-1}$.

O estádio fenológico de maior demanda hídrica foi o intermediário com 5,2 $\mathrm{mm}_{\text {dia }}{ }^{-1}$.

Os valores médios de $\mathrm{Kc}$ obtidos foram 0,39; 0,80; 1,14; 0,59 (Crimson Sweet); 0,35; 0,$72 ; 1,20 ; 0,30$ (Top Gun) e 0,34; 0,58; 0,89:0,55 (Shadow), para as fases inicial, vegetativa, intermediária e maturação, respectivamente.

\section{Referências}

Allen, R.G., Pereira, I.S., Raes, D., Smith. 2006. Evapotranspiracion del cultivo: guias para la determinación de los requerimientos de agua de los cultivos. FAO. Estudio Riego e Drenaje Paper, 56. Roma. 298 p.

Andrade Junior, A.S. de, Bastos, E.A., Silva, C.O. da, Gomes, A.A.N., Figueredo Júnior, I.G.M. de. 2004. Atlas Climatológico do Estado do Piauí. Embrapa Meio-Norte, Teresina, Brasil. 1 CD-ROM. (Embrapa Meio-Norte. Documentos, 101).

Bastos, E A., Silva, C.R., Rodrigues, B.H.N., Andrade Júnior, A.S. de, Ibiapina, L.M. M. 2007.
Coeficiente de cultivo da melancieira irrigada por gotejamento nos Tabuleiros Litorâneos, PI. In: Congresso Brasileiro de Agrometeorologia, Aracaju. Efeito das mudanças climáticas na agricultura. Anais... Campinas: Sociedade Brasileira de Agrometeorologia, 5 p. 1 CD-ROM.

Bezerra, F.M.L., Oliveira, C.H.C. de. 1999. Evapotranspiração máxima e coeficiente de cultura nos estádios fenológicos da melancia irrigada. Revista Brasileira de Engenharia Agrícola e Ambiental 3: 173-177.

Carlos, A.L.X., Menezes, J.B., Rocha, R.H.C., Nunes, G.H. de S., Silva, G.G. da. 2002. Vida útil pós-colheita de melancia submetida a diferentes temperaturas de armazenamento. Revista Brasileira de Produtos Agroindustriais 4: 29-35.

Carvalho, L.C.C. de, Bezerra, F.M.L., Carvalho, M.A.R. de. 2007. Evapotranspiração e coeficientes de cultivo da melancia sem sementes. Revista Ciência Agronômica 39: 53-59.

Dias, R. de C.S., Silva, C.M. de J., Queiroz, M.A. de, Costa, N.D., Souza, F. de F., Santos, M.H. dos, Paiva, L.B. de, Barbosa, G. da S., Medeiros, K.N. de. 2006. Desempenho agronômico de linhas de melancia com resistência ao oídio. Horticultura Brasileira 24:1416-1418.

Ferreira, J.O.P. 2010. Evapotranspiração e coeficientes de cultura da melancieira irrigada por gotejamento em Alvorada do Gurguéia-PI. $121 \mathrm{f}$. (Tese de Doutorado) - Universidade Estadual Paulista, Jaboticabal, Brasil.

IBGE. Sistema de recuperação automática Sidra: Produção agrícola Municipal: quantidade produzida, valor da produção, área plantada e área colhida da lavoura temporária. 2010. http://www.sidra.ibge.gov.br/< Acesso em 01 Fev. $2011>$

Miranda, F.R. de, Oliveira, J.J.G., Souza, F. 2004. Evapotranspiração máximas e coeficientes de cultivo para a cultura da melancia irrigada por gotejamento. Revista Ciência Agronômica 35: 36-46.

Rezende, G.M. de, Dias, R. de C.S., Costa, N.D. Cima. In: Dias, R. de C.S., Rezende, G.M. de, Costa, N.D. Sistema de produção de melancia. 2010. http://sistemasdeproducao. cnptia.embrapa.br/FontesHTML/Melancia/ SistemaProducaoMelancia/clima.htm/<Acesso em 23 Fev. 2012>

Santos, H.G. dos, Jacomine, P.K.T., Anjos, L.H. C. dos, Oliveira, V.A. de, Oliveira, J.B. de, Coelho, M.R., Lumbreras, J. F. 2006. Sistema Brasileiro de Classificação de Solos. Embrapa Solos, Rio de Janeiro, Brasil. 306 p.

Silva, C.R., Andrade Júnior, A.S., Melo, F.B., 
Sousa, A.B., Souza, C.F. Calibração da sonda de capacitância Diviner $2000^{\circledR}$ em um Argissolo. 2006. In: XXXV Congresso Brasileiro de Engenharia Agrícola. Resumos Expandidos ... João Pessoa, Brasil.

Syngenta Seeds, 2010. 'Top Gun®'. Folder. São Paulo, Performance Brasil.

Sousa, V.F, Coelho, F.E, Souza, B.A.V. 1999. Freqüência de irrigação em meloeiro cultivado em solo arenoso. Pesquisa Agropecuária Brasileira 34: 659-664.

Thornthwaite, C.W., Mather, J.R. 1955. The water balance. Drexel Institute of Technology, New Jersey, USA. $104 \mathrm{p}$. 